\title{
The prospects of using the silver nanoparticles composition in sodium alginate matrix
}

\author{
Anatoly Kubyshkin ${ }^{1}$, Olga Pisareva $^{1,{ }^{*}}$, Yevgenia Bessalova ${ }^{1}$, and Irina Fomochkina ${ }^{1}$ \\ ${ }^{1}$ V. I. Vernadsky Crimean Federal University, Medical Academy Named after S.I. Georgievsky, 5/7 \\ Lenina boul, Simferopol, 295051, Russia
}

\begin{abstract}
A wide range of anti-inflammatory properties of the solution of nanosilver has found its application in medical practice. In this work, nanosilver was used in the treatment of burn wounds in combination with a proteinase inhibitor. An experimental model of a second-degree burn with the use of a nano-silver solution in combination therapy is accompanied by a decrease in the inhibitory potential at the local level and stimulation of regeneration processes, which indicates the anti-inflammatory effects of nanosilver particles. There is also a decrease in the morphological signs of inflammation in the burned areas of the skin of the therapeutic use of a solution of nano-silver. The results indicate that nano-silver solution for prophylactic and therapeutic use has the ability to block a cascade of inflammatory reactions in the focus of damage, as it is evidenced by a decrease in the degree of alteration and intensification of reparative processes in damaged tissues.
\end{abstract}

\section{Introduction}

Since ancient times, the unique properties of silver, which were widely used in medicine, has been known. The study of the biological properties of metal nanoparticles in the context of nanopharmacology, as well as from the standpoint of nanotoxicology is currently the leading priority task of modern areas of science and technology [1, 2]. Modern advances in nanotechnology, including methods for producing nanomaterials, evaluating efficacy and safety, and using them for therapeutic and diagnostic purposes, are becoming one of the priority goals of modern materials science and medicine [3]. In connection with the mentioned above, the development and study of the mechanisms of action of silver nanoparticles compounds for the correction of various human body pathological conditions, including difficulties to treat by standard methods, is of particular interest [4].

According to a number of scientific studies, there is evidence that silver in the nanoscale range may have a pronounced bactericidal, virucidal effect, including having a broad antiseptic effect on pathogenic biota $[3,5]$. Due to the high specific surface of silver nanoparticles, the area of contact of silver nanoparticles with the bacterial cell wall increases, due to which an expressed bactericidal effect is manifested [6]. The intensive development of nanobiotechnology allows us to influence the structure of silver, changing it to the nanoscale range

\footnotetext{
Corresponding author: lolya.anat@gmail.com
} 
(with particle sizes up to $100 \mathrm{~nm}$ ), potentiating the biological effects of nanocompositions using silver nanoparticles.

At the same time, one of the urgent problems of modern medicine is timely and effective therapy for burn injuries of the skin; thermal burns occupy one of the leading places among all types of injuries: first of all, the dermis directly suffers, which plays an important role in the protective and immune reactions of the body [7]. An important role in the treatment tactics of burn injuries of the skin from thermal effects is taken by drug therapy, aimed primarily at accelerating reparative processes in damaged tissues $[8,9]$. To date, the list of drug supply with antioxidant, anti-inflammatory and reparative properties is quite large $[10,11]$. However, none of the standard treatment regimens is universal, and in certain cases there is a high percentage of complications in this category of patients associated both with infectious agents and the scarring, contractures etc.

Drugs in the form of ointments have rather effectively proven themselves in the complex therapy of burn injuries. A promising direction of nanopharmacology is the enhancement of the properties of drugs with the additional introduction of silver nanoparticles into these compositions $[12,13]$. Their combined use is relevant in view of the increased antibiotic resistance of microflora due to irrational antibiotic therapy and the recently increased multiresistant strains.

The most common theory of the physicochemical properties of silver nanoparticles regarding to their effect on a bacterial cell is the theory of "adsorption". It is based on the action of electrostatic forces between the cell wall of a microorganism, on which surface there is a negative charge, as well as silver ions, which have a positive charge when they are adsorbed between each other $[14,15]$. However, the possibility of using silver nanoparticles requires further preclinical, as well as clinical trials due to insufficiently studied their possible toxic effects on cells, tissues and organs, and body systems [16].

In this regard, the validation of the nanosilver solution developed at Taurida National University named after V.I. Vernadsky with the participation of employees of the Institute of Biology of the Southern Seas is relevant and timely. The composition of the solution includes: silver nanoparticles with a size of $10-20 \mathrm{~nm}(0.1 \%)$, sodium alginate $(0.6 \%)$ and distilled water (99.3\%). Currently, a number of studies have been carried out on the antimicrobial activity of silver nanoparticles, but their stabilization from the standpoint of toxicological safety, as well as the achievement of a satisfactory therapeutic effect with sodium alginate solution, has not been studied sufficiently [17]. In this regard, the purpose of the study is to investigate the effects of transdermal application of a solution of nano-silver on the burn skin surface of experimental animals.

\section{Materials and methods}

A series of experiments was carried out on white Wistar male rats weighing 180-210 g, in accordance with the principles of the European Convention for the Protection of Vertebrate Animals used for experimental or for other scientific purposes (Strasbourg, 1986). Experimental studies were carried out in aseptic conditions of the operating vivarium, the laboratory of the department of general and clinical pathophysiology, the department of pathological anatomy with a sectional course, and the histological laboratory with immunohistochemistry and electron microscopy of the Central Research Laboratory of the Medical Academy named after S.I. Georgievsky (structural unit) V. I. Vernadsky Crimean Federal University. The experiment was approved by the ethics committee (protocol No. 2 of September 11, 2015).

A preliminary series of experimental modeling of a burn allowed us to identify the most appropriate method for obtaining a burn in size and depth corresponding to the II degree. Simulation of a II degree burn was performed after intravenous administration of sodium 
thiopental, at the rate of $20 \mathrm{mg} / \mathrm{kg}$ weight. The burn was reproduced by application to the previously depilated skin area in the interscapular region, bounded by a wooden stencil $1.5 \times$ $1.0 \mathrm{~cm}$ in size, by a thin-walled glass container with water at a temperature of $90^{\circ} \mathrm{C}$ for $10 \mathrm{sec}$ [18]. Throughout the experiment, the damaged area of the skin remained open.

Experimental groups of animals were distributed as follows:

Group $1(\mathrm{~K})$ - intact animals $(\mathrm{n}=10)$.

Group 2 (G) - II degree burn model, observation for 7 days $(n=10)$.

Group $3(\mathrm{Y})$ - II degree burn model, treatment with antibiotic erythromycin for 7 days $(n=10) .0 .5$ g erythromycin was crushed in a porcelain mortar to a fine powder and mixed with $10 \mathrm{ml}$ of sodium alginate $(0.6 \%)$. Then $1 \mathrm{ml}$ obtained $10 \%$ solution was used for the local applications of a burn wound.

Group 4 (W) - II degree burn model, treatment with combination of nano-silver solution with the protease inhibitor aprotinin for 7 days $(n=10)$.

For the experiment, $3 \mathrm{ml}$ of Gordox (aprotinin) was mixed with $2 \mathrm{ml}$ of the original composition of nano-silver stabilized with sodium alginate $(0.1 \%$ nano-silver $)$, and then mixed with $5 \mathrm{ml}$ of nano-silver in the form of a gel $(0.1 \%$ nano-silver and $1.6 \%$ high molecular alginate sodium). Thus, the composition contained $30 \%$ of the aprotinin, $0.07 \%$ nano-silver, and $0.8 \%$ of a mixture of low and high molecular weight sodium alginate.

On the 7th day, a set of research methods was used: studies of the activity of nonspecific proteinases and their inhibitors in serum and supernatants of skin homogenates by enzymatic methods [19], macroscopic description, histological examination with hematoxylin and eosin staining, and picrofuchsin by van Gieson, transmission electron microscopy (TEM).

The animals were removed from the experiment under thiopental anesthesia, then euthanasia was performed and samples of the damaged skin area $1 \mathrm{~cm} \times 1 \mathrm{~cm} \times 1 \mathrm{~cm}$ were taken, followed by fixation in a $10 \%$ solution of neutral formalin to evaluate histological changes. To assess the histological changes at the tissue level, a skin fragment was fixed in $10 \%$ neutral formalin solution, followed by standard wiring and paraffin infiltration in a LOGOS processor (Italy) according to the manufacturer's recommendation. The use of a Leica DM2000 microscope (Germany) and a camera made it possible to obtain digital photographs of histological specimens. For the ultrastructural study, the pieces were fixed in a solution of glutaraldehyde, the sample preparation was performed manually, strictly following the protocol, and the PEM-125 transmission electron microscope (Sumy, Ukraine) was used for visualization.

\section{The results of the study and their discussion}

Modeling a thermal II degree skin burn demonstrates disorders in state of proteinase inhibitory system, both local and systemic level. Among the mediators of biochemical inflammation, nonspecific proteinases occupy the main positions due to their destructive influence on the skin structure. Thus, an increase in the level of activity of trypsin-like proteinases in the supernatants of skin homogenates was established at all stages of the study. The dynamics of changes in nonspecific proteinases and their inhibitors looks like a progressive proteolysis on the background of a severe exhaustion of the inhibitory potential, which leads to the development of the inflammatory process. Subsequently, a cascade of inflammatory reactions is initiated, resulting in proteolytic destruction of collagen fibers, histohematogenous barriers, edema and hemorheological changes at the level of microcirculation, including acute decrease and damage of reparative properties in alterized tissues. It is important to monitor the potential of pharmacological correction, as well as the dynamics of changes of protease-inhibitory system at the local level. Functional indicators of proteinase inhibitory system can be considered as a criterion for the effectiveness of the proposed selected nanofarmacotherapy. 
Considering the results of histological examination of damaged skin areas, it was found that the main morphological changes are exudative-necrotic foci and impaired hemodynamics. On the 7th day, the burn area is covered with a scab, which begins to partly reject, and at its border with the underlying tissues there is a rather wide zone of demarcation inflammation with a prevalence of neutrophilic leukocytes in the exudate. Inflammatory infiltration extends to the deep layers of the dermis, in some areas penetrating into the subcutaneous fat. Plots of granulation tissue begin to appear at the bottom and edges of the burn wound. Hemodynamic disorders are presented in the form of pronounced plethora, edema, small hemorrhages. There is a slight increase in the manifestations of edema in the deeper parts of the dermis (Fig. 1A).

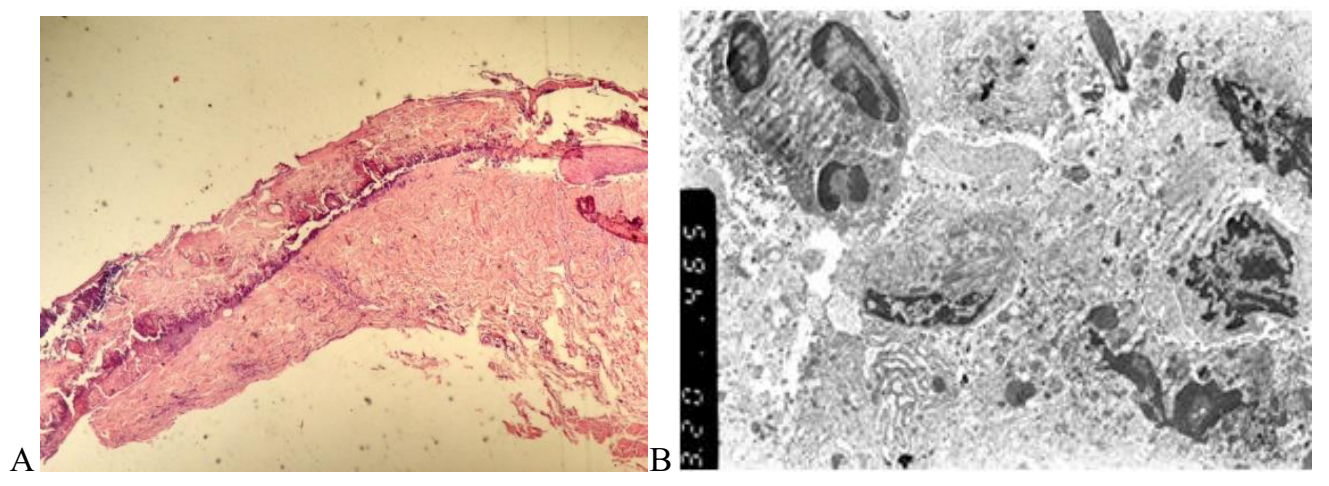

Fig. 1. A.Thermal burn. Paraffin sections, hematoxylin and eosin stain. 7th day, 40x. B. Ultrathin cuts. Transmission electron 7 th day. 5800x.

According to electron microscopy, edema, leukocyte-macrophage infiltration and activation of cells of the fibroblastic differentia are the most demonstrative (Fig. 1B). On the 7th day, the damaged areas of the skin are infiltrated with lymphocytes, actively phagocytic macrophages and degranulating neutrophilic granulocytes. Multiple invaginations in lymphocyte nuclei, central arrangement of the nucleolus and marginal heterochromatin are signs of their active state. Fibroblasts are few, have an elongated shape, small processes, and revealed a large number of free ribosomes in their cytoplasm. Intracellular edema and granular dystrophy are characteristic of most cells in the dermis. Expansions of the granular endoplasmic reticulum take the form of bag-like elongated cavities separated by thin sections of the cytoplasm with fine-grained contents. Mitochondria of irregular polygonal shape, densely filled with laminar cristae, have a dark matrix. In the extracellular space, a small amount of collagen fibrils is noted, amorphous substance prevails.

In groups with drug correction, the following pathological changes occurred: on the 7 th day the scab looks uneven, there are areas of partial scab rejection with the formation of blisters containing exudate. Granulation tissue with multiple fibrils and active fibroblasts significantly developed. Demarcation inflammation is represented by diffuse focal lymphatic macrophage infiltration spreading to the underlying tissues. On the periphery of the burn zone and in the tissues of the dermis there are signs of spasm, edema, plethora, a small amount of hemorrhages. The epithelium at the edges of the wound is hyperplastic, with symptoms of acanthosis, hyper- and parakeratosis. The growth of granulation tissue and the formation of young connective tissue are noted (Fig. 2A). 

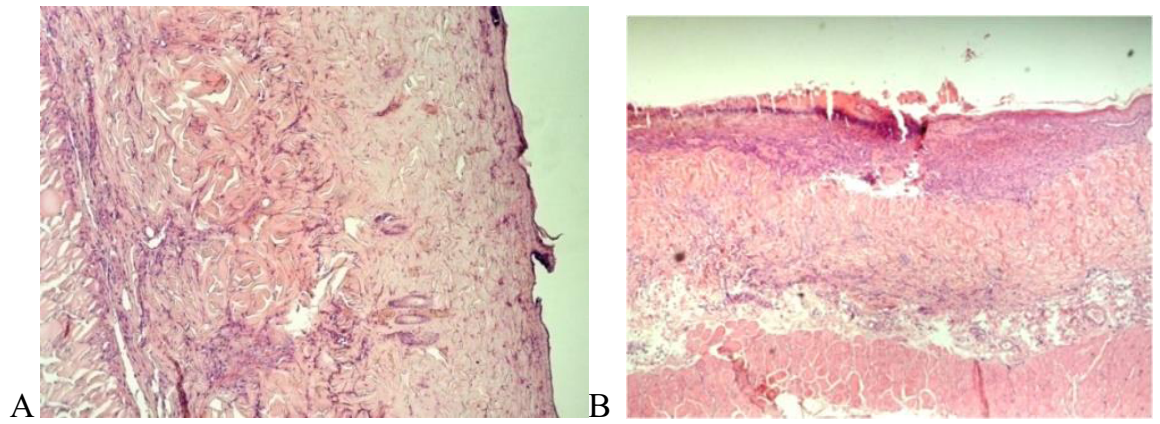

Fig. 2. Fragment of the damaged skin area with the use of an antibiotic 40x. B.Fragment of the damaged skin area using nanofarmacocorrection 100x. Paraffin cut, stained with hematoxylin and eosin.

In the group receiving the combined nanofarmacocorrection (nano-silver + aprotinin), on the 7th day there is a thinning and rejection of the scab over large areas, epithelialization of the wound surface is pronounced to a large extent and spreads almost to the center of the damaged skin area (Fig. 2B). A large amount of granulation tissue with fine connective tissue fibers was detected in the visual fields. Instead of a random arrangement of fibers, their predominantly horizontal direction is noted, parallel to the surface of the epidermis. The layer of granulation tissue in the center is thicker, only as it approaches the edges it becomes thinner. Hemodynamic changes are reduced, as well as inflammatory infiltration in the damaged area.

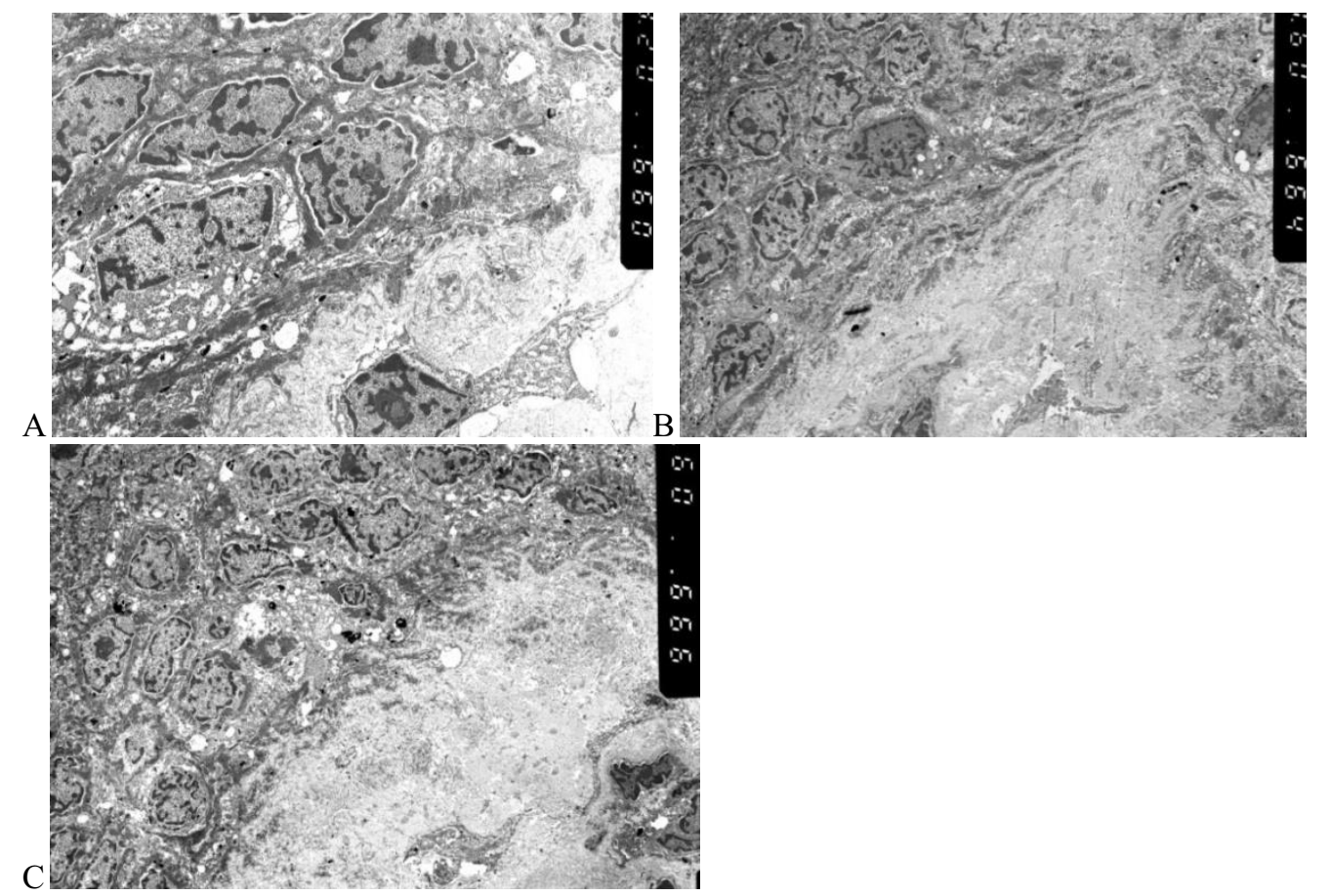

Fig. 3. A. Fragments of the epidermis and dermis of the skin in case of burn correction with erythromycin solution for 7 days. TEM. 5000x. Fragments of the epidermis and dermis (A) and the area of pronounced proliferation of the epidermis (B) of the skin during the drug correction of the burn by the combined method (nano-silver + aprotinin) for 7 days. TEM. 4000x.

When conducting electron microscopy, it revealed a similar dynamics of changes at the ultrastructural level. With the correction of burn with erythromycin, there is a good 
preservation of all skin structures (Fig. 3A). Kernels of keratinocytes have shallow invaginations of the karyolemma. The perinuclear space of most cells is slightly expanded and continues into the cisterns of the granular endoplasmic reticulum (GER) surrounding the nucleus. Ribosomes, polysomes, and also fragments of GER cisterns are located in the perinuclear zone of the cytoplasm. A large number of mitochondria, small, most of them contain a dark matrix and a large number of cristae. In some fields of view there are cells that have a blade nucleus, small and medium-sized vacuoles, formed as a result of the expansion of the GER cistern and the Golgi complex (Fig. 3A). It was established that the revealed structure of keratinocytes reflects the active synthetic activity of cells. The capillaries of the dermis have a thickened basement membrane, endotheliocytes are intact. A thicker layer of connective tissue is formed by circular fibers forms around the vessels (Fig. 3A). Some cells have a normal ultrastructure of the nucleus and cytoplasmic organelles. In another part of the fibroblasts, hydropic changes were noted with the formation of vacuoles as a result of the swelling of mitochondria, the expansion of the GER cistern and the Golgi complex.

In the experimental group with the use of combined treatment using nanosilver, the best preservation of the skin structures was found: keratinocytes, fibroblasts, dermis vessels. Intercellular contacts and complexes of tonofilaments with desmosomes are restored (Fig. 6 B). The degree of vacuolization is reduced, but retained (Fig. $6 \mathrm{C}$ ). In keratinocytes, the ultrastructure is not changed (Fig. 6 B). Kernels contain numerous invaginations. Euchromatin is predominant, and heterochromatin in the same amount is represented by small-size karyosomes and marginal heterochromatin. In the hyaloplasm, a significant number of polisomes are found. The cytoplasm of cells contains a huge number of mitochondria with a preserved structure, a dark matrix, a large number of cristae. In the growth layers of the epidermis, there are single young cells with a large lobar nucleus, moderate depletion of organelles (Fig. 6C). The appearance of such keratinocytes can be considered as a result of the activation of reparative processes. In the papillary layer of the dermis there are groups of differentiated fibroblasts with signs of the functional activity of synthetic processes.

Thus, the conducted studies allowed demonstrating a number of positive properties of nanofarmacotherapy in the treatment of burns of the skin, in more detail the properties of silver nanoparticles are shown in the diagram (Fig. 4).

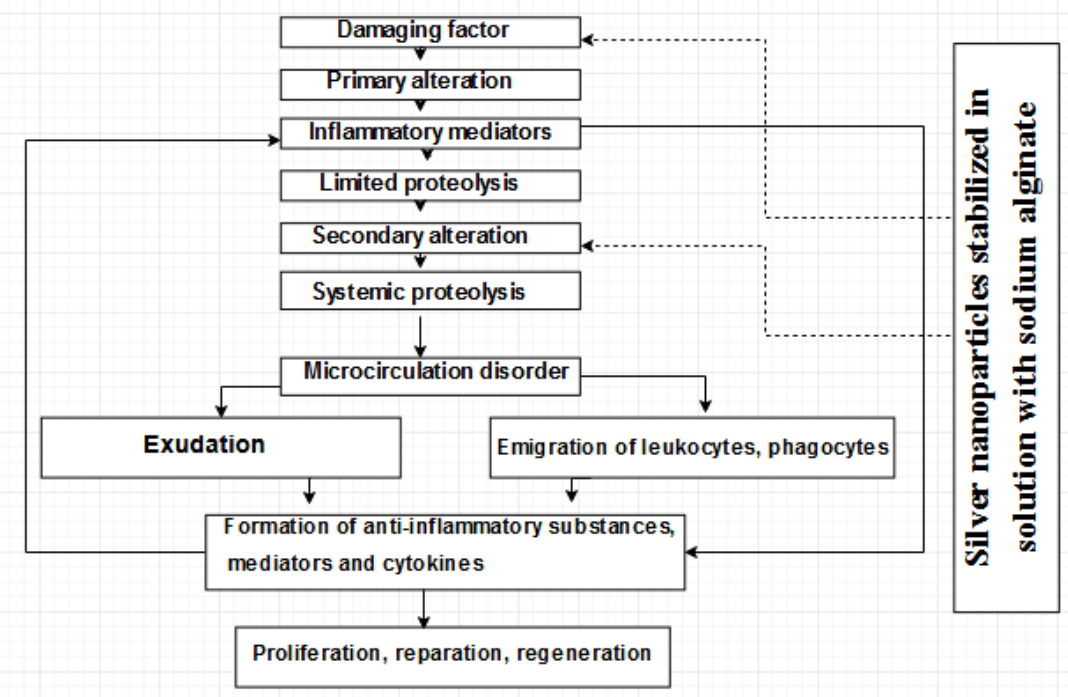

Fig. 4. Pathogenetic scheme of pharmacological use of silver nanoparticles in the development of the inflammatory process. 


\section{Conclusion}

In this work, it is proved that the imbalance in the system of nonspecific proteases and their inhibitors at the local level is characterized by activation of proteases against the background of depletion of the inhibitory potential, which indicates the pathogenetic expediency of using drugs of the protease inhibitor group as a local treatment of thermal burn skin damage. Along with the use of the proteinase inhibitor Aprotinin, we should not forget about the important pathogenetic significance of the nano-silver particle solution, since it has a number of properties necessary for treating thermal skin burns: antimicrobial activity (reduction of infectious complications) does not reduce the processes of local immune reactivity, accelerates tissue regeneration processes. That is why the most effective combination for experimental correction is a combination in the form of applications with a solution of nano-silver and aprotinin.

Based on the above experimental data on animal models (white rats-males of the Wistar line), the efficacy and feasibility of using combined drugs based on silver nanoparticles in the presence of a wide spectrum of bactericidal, bacteriostatic, virulocidal, fungicidal, antiseptic, including the number of activators of reparative processes of damaged areas of the skin after thermal effects of extreme temperatures. Thus, thanks to the achievements of nanotechnology, in particular nanopharmacology and nanotoxicology, one of the priority directions of modern medicine and nanocomposite production technology, one of the leading directions at present is the development, study of the effectiveness and safety of the application of the abovementioned advanced treatment methods. This therapy will reduce and adjust the qualitative and quantitative composition of treatment regimens, optimize, reduce the risk of the likelihood of side effects, prevent and prevent the development of antibiotic resistance and the emergence of new multiresistant microorganism strains, reduce the number of complications and adverse outcomes in patients with this pathology.

\section{References}

1. E. M. Egorova, A. A. Kubatiev, V. I. Shvec, The science, .6 (74), p.350 (2014)

2. M. V. Taran, N. F. Starodub, A. M. Katsev et al., Biophotonics, 9032, 1-7 (2013)

3. I. S. Chekman, B. A. Movchan, M. I. Zagorodnyj, The art of healing, 51(5), 32-34 (2008)

4. T. M. Bojchuk, N. J. Andrijchuk, L. I. Vlasyk, Clinical and experimental pathology, 4(42), 151-157, (2012)

5. M. Amin, F. Anwar, M. Ramzan Saeed Ashraf Janjua et al., Int. J. Mol. Sci., 13, 99239941 (2012)

6. A. I. Gusev, Fizmatlit, 2, 414 (2007)

7. A. N. Guillory, R. P. Clayton, D. N. Herndon, Int. J. of Molecular Sciences, 17, 53-53 (2016)

8. A. J. Singer, R. A. Clark, The New England Journal of Medicine, 3(41), 738-746 (1999)

9. L. K. Branski, A. Al-Mousawi, H. Rivero, M. G. Jeschke, A. P. Sanford, D. N. Herndon, Surgical Infections(Larchmt), 10(5), 389-397 (2009)

10. A. A. Alekseev, A. E. Bobrovnikov, S. B. Bogdanov, L. I. Budkevich, M. G. Krutikov, YU.I. Tyurnikov, All-Russian public organization "Association of Combustiologists". "A world without burns", 1-12 (2015)

11. V. Singh, L. Devgan, S. Bhat, S. M. Milner, Annals of Plastic Surgery, 59, 109-115 (2007)

12. T. V. Surakova, N. Yu. Zhidomorov, T. R.Grishina, A. A. Kodin, Russian medical Journal, 20(11), 575-581 (2012)

13. I. N. Yurkova, D. A. Panov, V. I. Ryabushko, Scientific notes of Taurida National University. V. I. Vernadsky, Series “Biology, Chemistry”, 22 (61), 203-207 (2009) 
14. E. G. Akopova, A. H. Kade, E. F. Kurnosenkova, Kuban Scientific Medical Bulletin, 1, 8$11(2007)$

15. E. K. Baranova, A. A Revina, L. I. Vojno, Materials of the 1st Russian Scientific and Methodological Seminar, 1, 53-60 (2003)

16. V. F. Moskalenko, L. G. Rozenfel'd, B. O. Movchan, Human and medicine. Materials of the I National Congress, 1, 167-168 (2008)

17. G. V. Odegova, V. A. Burmistrov, P. P. Rodionov, The use of silver drugs in medicine, 5, $53-60(2004)$

18. A. A. Mironov, Ya.Yu. Komissarchik, V.A. Mironov, The science J., 990, (1994)

19. A. V. Kubyshkin, V. Z. Kharchenko, P. F. Semenets L. L. Aliev, I. I. Fomochkina, L. V. Anisimova, Methods of determining the activity of non-specific proteinases and their inhibitors in blood and biological liquids (Methodical recommendation, Kiev, 2010) 